\title{
A Membrane-Based Flow-through Hybridization Technology: A Rapid and Versatile tool for Molecular Diagnostics
}

\author{
K.F.J. Chow*
}

Diagcor Bioscience Inc. Limited, Hong Kong SAR (China)

\begin{abstract}
Genetic diseases remain an important cause of ailment, morbidity and death for children and adults. Despite the recent advances of tools for molecular diagnosis such as DNA chips and protein arrays which are capable of analyzing many genes, SNPs and proteins, few of these systems have been applied in clinical diagnosis because the apparatus are still expensive and many of the genetic tests are less likely to be developed commercially. Hence most genetic tests are still being performed through conventional methods such as Dot-Blot hybridization and Slot-blot hybridization in clinical laboratories worldwide. The expensive, time consuming and labor-intensive nature of these tests make them unaffordable for popular use. The availability of a low-cost diagnostic system will make molecular testing cost efficient and become readily available worldwide. In this report, we evaluated the Flow-through hybridization system. Our results show that Flow-through hybridization method produces higher sensitivity in 5 to 15 minutes compared to those obtained through conventional hybridization in hours to overnight respectively without sacrificing specificity. An apparatus can also be readily adopted for membrane-based home-brew tests, $R \& D$ development as well as other commercially available diagnostic kits, hence making the Flow-through method a useful tool for molecular diagnosis.
\end{abstract}

\section{INTRODUCTION}

Advances in recombinant DNA technologies have facilitated the establishment of genomic sequences, the functional and regulatory systems of genes and protein leading to definitive information on genetic origins of life. The information lying within defective genes and their products from inheritance, sporadic mutations and pathogens can be readily detected. Hence molecular diagnostics have enjoyed a very rapid growth recently and shall soon become the effective tool for "Preventive Medicine". Undoubtedly, Microchip technology has revolutionized the way we study genomes and proteomes because of its ability to detect thousands of sequences, proteins or other analytes simultaneously. However it has been proven difficult to apply to routine clinical diagnosis and the complexity nature of high density chips will remain difficult to be validated. Moreover, the expensive instruments will only be confined in well-funded laboratories and research institutions where they can be afforded. In practice, definitive diagnosis is possible using hundreds or less sequences or analytes deduced from available research data. Thus "low density chip" is the ideal diagnostic tool because it is much more affordable, efficient and effective for product development worldwide. The ability to amplify a single copy into millions in a short period of time by PCR or equivalent method is the key for the advances in DNA analyses. However, the possibility of false amplification requires follow-up analysis for validation. Conventional analysis methods like fragment analyses or hybridization cannot provide adequate means for analysis either because of their specificity or throughput i.e. the ability to simultaneously analyse multiple targets or generating definitive results. Membrane hybridization is first developed by Southern and

*Address correspondence to this author at the Diagcor Bioscience Inc. Limited, Hong Kong SAR (China); E-mail: joseph@diagcor.com subsequent modification to Reversed Dot Blot (RDB) advanced by Maggio [1]. These techniques are capable of differentiating single base mutations and the array technology eliminate the difficulty of getting highly specific results for multiple analyses in a single run. Hence coupled with stringent hybridization conditions, the highest sensitivity and specificity can be achieved easily. The remaining focus should therefore be on the speed of hybridization, and the efficient use of the analytes and reagents without the use of sophisticated instrumentations. We would like to present the membrane-based "Flow-through Hybridization Platform" (US Patent number 5741647) which is capable of developing low cost membrane-based macro-arrays for use in molecular diagnostic applications. To illustrate the superiority of the Flow-through hybridization over conventional hybridization and its usefulness in diagnostics. Parallel assays on genotyping of multiple human papillomavirus (HPV) in "low-density array" format are used as examples.

\section{MATERIAL AND METHODS}

\section{Generation of Recombinant HPV DNA Positive Controls}

HPV genotypes 52 DNA has been cloned and verified by DNA sequencing. The cloned DNA was amplified by PCR using 5'-biotinylated labeled MY09/11 consensus primers as described previously [2,3] for subsequent hybridization. A pair of primers that amplified the $\beta$-globin gene was also included as a PCR control. In brief, 1 femtogram of HPV cloned DNA and 100ng of purified human genomic DNA was amplified in a $25 \mu \mathrm{l}$ PCR reaction mixture contain $10 \mathrm{mM} \mathrm{KCl}, 10 \mathrm{mM}$ Tris- $\mathrm{HCl}, 6 \mathrm{mM} \mathrm{MgCl}, 200 \mu \mathrm{M}$ dNTP, 250 pmol of each biotin-labeled primers and $2 \mathrm{U}$ of AmpliTaq Gold polymerase. Amplification was carried out in a thermal cycler (PE9700, Perkin-Elmer) with activation of AmpliTaq Gold polymerase at $95^{\circ} \mathrm{C}$ for $11 \mathrm{~min} ; 40$ cycles of denaturation at $95^{\circ} \mathrm{C}$ for $20 \mathrm{sec}$, annealing at $55^{\circ} \mathrm{C}$ for $30 \mathrm{sec}$, 
and extension at $72^{\circ} \mathrm{C}$ for $30 \mathrm{sec}$, and a final extension at $72^{\circ} \mathrm{C}$ for $5 \mathrm{~min}$. Each PCR product was verified by electrophoresis on $1.5 \%$ agarose gel stained with ethidium bromide for the presence of amplicons.

\section{Preparation of Membrane Array}

The membrane arrays were prepared through established standard operating procedures. Each array contained a total of 6 different high risk HPV type-specific oligonucleotide sequences probes, a PCR confirmation probes against human $\beta$-globin gene and internal hybridization control designed according to [4] (Fig. 1). The 5'-ends amino-modified oligo probes were dotted onto the Biodyne $\mathrm{C}$ membranes as described in [5]. The Flow-through and conventional hybridization assays were carried out with the same lot of membrane arrays, amplified PCR products and reagents in parallel to ensure the validity of comparison.

\section{Flow-through Based Reverse Dot Blot Hybridization}

Twenty microlitres of PCR product were denatured and incubated with $0.5 \mathrm{ml}$ of hybridization buffer (2X SSC, $0.1 \%$ SDS) at $41^{\circ} \mathrm{C}$ for 1 to 15 minutes according to Table $\mathbf{1}$. The membranes were washed three times with $0.75 \mathrm{ml}$ of hybridization buffer and blocked with $0.5 \mathrm{ml}$ of blocking buffer at $25^{\circ} \mathrm{C}$ for 5 minutes. $0.5 \mathrm{ml}$ of diluted alkaline phosphatase conjugate (BioRad) was added and incubated at $25^{\circ} \mathrm{C}$ in the device for 3.5 minutes. Unbound conjugate molecules were removed by three direct washes with $0.75 \mathrm{ml}$ of washing buffer $\left(0.1 \mathrm{M} \mathrm{NaCl}, 0.1 \mathrm{M}\right.$ Tris- $\mathrm{HCl}, 3 \mathrm{mM} \mathrm{MgCl}_{2}, 0.5 \%$ Tween $20 \mathrm{v} / \mathrm{v}, \mathrm{pH}$ 7.5) and colour development was performed by incubating $0.5 \mathrm{ml}$ of NBT/BCIP substrate (BD Biosciences) at $37^{\circ} \mathrm{C}$ for 8 minutes and the reaction was stopped by the addition of $0.5 \mathrm{ml}$ of TE. Membranes were rinsed three times with $0.75 \mathrm{ml}$ of ambient washing buffer. Developed membranes were interpreted within 5 minutes after NBT/BCIP substrate incubation. All steps of hybridization, washing and color development were completed in the R2-M Flow-through system (DiagCor Bioscience Incorporation Limited) by simply pipetting the corresponding solutions into respective reaction chamber wells, followed by built-in pump removal. Since the reaction chamber is accurately maintained at an appropriate temperature together with solutions of controlled ingredients to provide suitable stringency, highly specific and reproducible results were achieved.

\section{Conventional Reverse Dot Blot Hybridization}

The conventional reverse dot blot hybridization was assay in a manner similar to that described in the Flow-through hybridization assay. All primer and probe sequences were the same as those used in the Flow-through assay format. To compare the results between Flow-through and conventional hybridization, different incubation times for the conventional method were compared as shown in Table 1. The volume of the hybridization solution was carried out as described in Table 4 in a water bath and all tests were performed in triplication.

\section{Data Analysis}

To standardize the result interpretation and minimize any subjective analysis by visual interpretation, all membranes were scanned using a flatbed scanner (CanoScan LiDe60) at 300 dpi resolutions and analyzed by computer software (GenoFlow HPV Identifier Version 1.0, DiagCor Bioscience Incorporation Limited). The signal intensities and the background noise were transformed into numerical values for comparison. All signal intensities were normalized with the internal standard located on each membrane and the Z-score (standard score) obtained (Table 2).

A

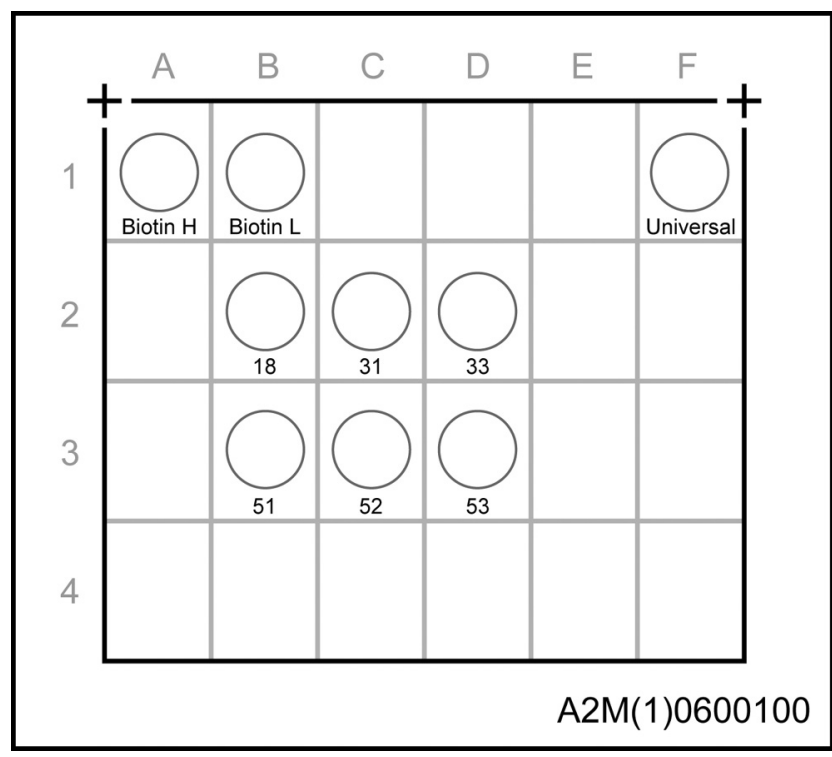

B

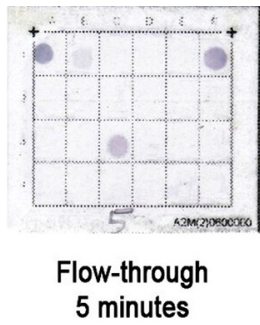

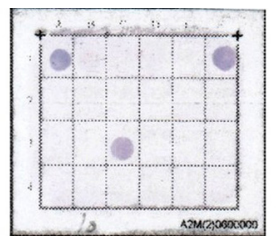

Flow-through 10 minutes

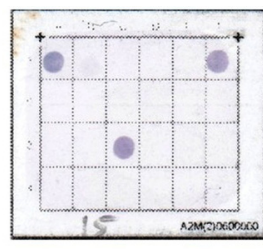

Flow-through 15 minutes
C

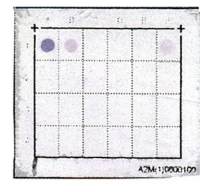

Conventional Hybridization 5 minute

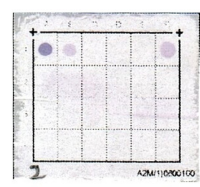
10 minute
Conventional Hybridization

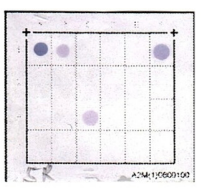

Conventional Hybridization 120 minute

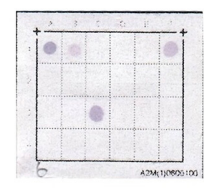

Conventional Hybridization Over Night
Fig. (1). (A) Schematic diagram of the membrane probed with different oligonucleotide probes for twenty two different HPV types. Two biotin controls and a PCR amplification control, act as the internal reaction control for each hybridization reaction. (B) Results of membrane by Flow-through method hybridizing at different time. (C) Results of Conventional Method hybridizing at different hybridization incubation. The result of 5-min hybridization using the flow-through method is comparable to that of 2-hour hybridization by the conventional method. A 10-minute hybridization by the flow-through can achieve the same signal produced by an overnight hybridization using the conventional method. And the flow-through hybridization method produced a slight decrease (although not significant) in the background noise. 
Table 1. Experimental Setting in the Flow-through and the Conventional Method Respectively. Different Hybridization Duration was Used and Compared in the Two Methods

\begin{tabular}{|c|c|c|c|c|c|c|c|c|}
\hline Test & $\begin{array}{c}\text { Pre- } \\
\text { hybridization }\end{array}$ & Hybridization & $\begin{array}{c}\text { Stringency } \\
\text { Wash }\end{array}$ & Blocking & $\begin{array}{c}\text { Enzyme } \\
\text { Conjugation }\end{array}$ & $\begin{array}{c}\text { Post-Reaction } \\
\text { Wash }\end{array}$ & $\begin{array}{c}\text { Color } \\
\text { Develop }\end{array}$ & $\begin{array}{c}\text { Stop } \\
\text { Reaction }\end{array}$ \\
\hline \multicolumn{9}{|c|}{ Time required (minutes) } \\
\hline \multicolumn{9}{|c|}{ Conventional Hybridization Method } \\
\hline 1 & $15 \mathrm{~min}$ & $5 \mathrm{~min}$ & $15 \min x 3$ & $15 \mathrm{~min}$ & $10 \mathrm{~min}$ & $15 \min \times 3$ & $8 \mathrm{~min}$ & $10 \mathrm{~min}$ \\
\hline 2 & $15 \min$ & $10 \mathrm{~min}$ & $15 \min \times 3$ & $15 \mathrm{~min}$ & $10 \mathrm{~min}$ & $15 \mathrm{~min} \times 3$ & $8 \mathrm{~min}$ & $10 \mathrm{~min}$ \\
\hline 3 & $15 \mathrm{~min}$ & $30 \mathrm{~min}$ & $15 \min x 3$ & $15 \mathrm{~min}$ & $10 \mathrm{~min}$ & $15 \min \times 3$ & $8 \mathrm{~min}$ & $10 \mathrm{~min}$ \\
\hline 4 & $15 \min$ & $60 \mathrm{~min}$ & $15 \min x 3$ & $15 \mathrm{~min}$ & $10 \mathrm{~min}$ & $15 \min \times 3$ & $8 \mathrm{~min}$ & $10 \mathrm{~min}$ \\
\hline 5 & $15 \min$ & $120 \mathrm{~min}$ & $15 \min x 3$ & $15 \mathrm{~min}$ & $10 \mathrm{~min}$ & $15 \mathrm{~min} \times 3$ & $8 \mathrm{~min}$ & $10 \mathrm{~min}$ \\
\hline 6 & $15 \min$ & $720 \mathrm{~min}$ & $15 \min \times 3$ & $15 \mathrm{~min}$ & $10 \mathrm{~min}$ & $15 \min \times 3$ & $8 \mathrm{~min}$ & $10 \mathrm{~min}$ \\
\hline \multicolumn{9}{|c|}{ Flow-through Hybridization Method } \\
\hline 7 & 0 & $1 \mathrm{~min}$ & $1 \min x 3$ & $5 \mathrm{~min}$ & $3.5 \mathrm{~min}$ & $1 \min \times 3$ & $8 \mathrm{~min}$ & $1 \mathrm{~min}$ \\
\hline 8 & 0 & $2 \mathrm{~min}$ & $1 \min x 3$ & $5 \mathrm{~min}$ & $3.5 \mathrm{~min}$ & $1 \min x 3$ & $8 \mathrm{~min}$ & $1 \mathrm{~min}$ \\
\hline 9 & 0 & $3 \mathrm{~min}$ & $1 \min x 3$ & $5 \mathrm{~min}$ & $3.5 \mathrm{~min}$ & $1 \min x 3$ & $8 \mathrm{~min}$ & $1 \mathrm{~min}$ \\
\hline 10 & 0 & $4 \mathrm{~min}$ & $1 \min x 3$ & $5 \mathrm{~min}$ & $3.5 \mathrm{~min}$ & $1 \min x 3$ & $8 \mathrm{~min}$ & $1 \mathrm{~min}$ \\
\hline 11 & 0 & $5 \mathrm{~min}$ & $1 \min x 3$ & $5 \mathrm{~min}$ & $3.5 \mathrm{~min}$ & $1 \min x 3$ & $8 \mathrm{~min}$ & $1 \mathrm{~min}$ \\
\hline 12 & 0 & $6 \mathrm{~min}$ & $1 \min x 3$ & $5 \mathrm{~min}$ & $3.5 \mathrm{~min}$ & $1 \min x 3$ & $8 \mathrm{~min}$ & $1 \mathrm{~min}$ \\
\hline 13 & 0 & $8 \mathrm{~min}$ & $1 \min x 3$ & $5 \mathrm{~min}$ & $3.5 \mathrm{~min}$ & $1 \min x 3$ & $8 \mathrm{~min}$ & $1 \mathrm{~min}$ \\
\hline 14 & 0 & $10 \mathrm{~min}$ & $1 \min x 3$ & $5 \mathrm{~min}$ & $3.5 \mathrm{~min}$ & $1 \min x 3$ & $8 \mathrm{~min}$ & $1 \mathrm{~min}$ \\
\hline 15 & 0 & $15 \mathrm{~min}$ & $1 \min x 3$ & $5 \mathrm{~min}$ & $3.5 \mathrm{~min}$ & $1 \min x 3$ & $8 \mathrm{~min}$ & $1 \mathrm{~min}$ \\
\hline
\end{tabular}

\section{RESULTS}

Comparison of the Flow-through with Conventional Hybridization in Terms of Sensitivity and Time Required

In the present study, detection of human papillomavirus was taken as a model to demonstrate the sensitivity of the Flow-through hybridization compared to conventional hybridization. One femtogram of the HPV plasmid DNA was amplified by PCR reaction for testing the Flow-through and the conventional hybridization method at different hybridization time stages. With the Flow-through process, a detectable signal was observed with an incubation time of 1 minute. There was a sharp increase in the signal intensity in the first ten minutes in the Flow-through method. It has also demonstrated that with 5 minutes of the Flow-through hybridization process, $71 \%$ of the signal intensity was achieved in comparison to an overnight conventional method. With 8-10 minutes incubation, Flow-through hybridization was able to achieve similar sensitivity in conventional overnight hybridization. Therefore, Flow-through method was able to reduce hybridization time by 25-100 folds (Table 3). In addition, the Flow-through process showed to have an increased sensitivity compared to the conventional method with extended hybridization time to 15 minutes. A higher signal was detected as compared to the overnight conventional signal. Thus the
Flow-through process was shown to be superior over the conventional process by way of shortening the hybridization time as well as giving a higher sensitivity.

\section{Overall Processing Time, Reagents, Consumption and Cost}

It is understood that by using a much smaller reaction surface area in the case of the Flow-through process, substantial savings in reagents can be achieved. The processing time and reagent volume used were compared and depicted in Table $\mathbf{3}$ and $\mathbf{4}$. In a comparable intensity assay pair, such as the 5-minute Flow-through hybridization compared to the 120-minute conventional hybridization, the overall time reduction was 10 -fold. The overall volume of reagent was reduced by 12.5 times in the Flow-through method and the overall cost was estimated to be reduced by 8.5 folds (Table 4). Evidently an overall reduction by 9.6 folds including technical skilled time would be a major saving can be transformed into higher productivity or profitability. Furthermore, with a substantial increase in sensitivity in the Flow-through process was obtained through the use of less reagents to produce comparable or even better signals (Fig. 2). This is very important for diagnosis because reporting time is often the limiting factor. 
Table 2. Hybridization Results in Terms of Z-Score. Z-Score was Transformed into Numerical Value (Signal-to-Noise Ratio of the Target Signal Normalized by the Internal Control) for Comparison. Standard Overnight Conventional Hybridization was Used as a Standard for Signal Calculation

\begin{tabular}{|c|c|c|c|c|}
\hline $\begin{array}{l}\text { Hybridization } \\
\text { Time (min) }\end{array}$ & Z-Score & $\begin{array}{l}\text { Percentage with Respective to Overnight } \\
\text { Conventional Signal Intensity }\end{array}$ & Z-Score & $\begin{array}{l}\text { Percentage with Respective to Overnight } \\
\text { Conventional Signal Intensity }\end{array}$ \\
\hline 1 & 0.2540 & $29 \%$ & $\mathrm{nt}^{a}$ & - \\
\hline 2 & 0.3278 & $37 \%$ & $\mathrm{nt}$ & - \\
\hline 4 & 0.3047 & $35 \%$ & $\mathrm{nt}$ & - \\
\hline 5 & 0.6253 & $71 \%$ & 0.0861 & $10 \%$ \\
\hline 6 & 0.4694 & $53 \%$ & $\mathrm{nt}$ & - \\
\hline 30 & 1.2356 & - & 0.3106 & $35 \%$ \\
\hline 60 & $\mathrm{nt}$ & - & 0.3916 & $44 \%$ \\
\hline 120 & $\mathrm{nt}$ & - & 0.6375 & $72 \%$ \\
\hline $720^{b}$ & $\mathrm{nt}$ & - & 0.8810 & $100 \%$ \\
\hline
\end{tabular}

${ }^{\mathrm{a}} \mathrm{nt}=$ Not tested

${ }^{b} 720$ minutes taken as a reference to an overnight hybridization.

Table 3. Comparison of Total Hybridization Time Required for Similar Signal Produced. Two Different Sets of Hybridization time (Flow-through 5 Minutes vs Conventional 120 Minutes; Flow-through 10 Minutes vs Conventional 720 Minutes) were Used for Comparison

\begin{tabular}{|c|c|c|c|c|}
\hline Method & Flow-through (Min) & Conventional (Min) & Flow-through (Min) & Conventional (Min) \\
\hline Pre-hybridization & 0 & 15 & 0 & 15 \\
\hline Hybridization & 5 & 120 & 10 & 720 \\
\hline Blocking & 5 & 15 & 5 & 15 \\
\hline Enzyme Conjugation & 3.5 & 10 & 3.5 & 10 \\
\hline Conjugate wash (3 times) & 3 & 45 & 3 & 45 \\
\hline Color development & 8 & 8 & 8 & 8 \\
\hline Total Time & 31.5 & 308 & 36.5 & 908 \\
\hline
\end{tabular}

\section{DISCUSSION}

Nucleic acid hybridization has been one of the most widely used methods in researches and in clinical diagnostics applications. Over the past decade, DNA analysis has expanded enormously due to the advances in new scientific knowledge and analytical technology. Because of the expo- 
Table 4. Comparison of Reagent Volume and Cost in Flow-through and Conventional Hybridization. Calculation of cost, Reagent Volume Used in Each Step and Technical Time of the Two Hybridization were Based on 5 Minutes Flow-through and 120 Minutes Conventional Hybridization

\begin{tabular}{|c|c|c|c|c|}
\hline Hybridization & $0.5 / \$ 0.1$ & $5 / \$ 1$ & $10 x$ & $10 x$ \\
\hline Post-hybridization Wash (3 times) & $2.25 / \$ 0.45$ & $30 / \$ 6$ & $13 x$ & $13 x$ \\
\hline Enzyme Conjugation & $0.5 / \$ 0.8$ & $5 / \$ 8$ & $10 \mathrm{x}$ & $10 \mathrm{x}$ \\
\hline Conjugate wash ( 3 times) & $2.25 / \$ 0.23$ & $30 / \$ 3$ & $13 x$ & $13 x$ \\
\hline Color development & $0.5 / \$ 0.7$ & $5 / \$ 7$ & $10 x$ & $10 x$ \\
\hline Post-reaction Wash (3 times) & $2.25 / \$ 0.23$ & $30 / \$ 3$ & $13 x$ & $13 x$ \\
\hline Technical time per run / Cost & 31.5 Mins / \$31.5 & 308 Mins / \$308 & - & $9.6 x$ \\
\hline
\end{tabular}

Conventional vs Flow-through hybridization

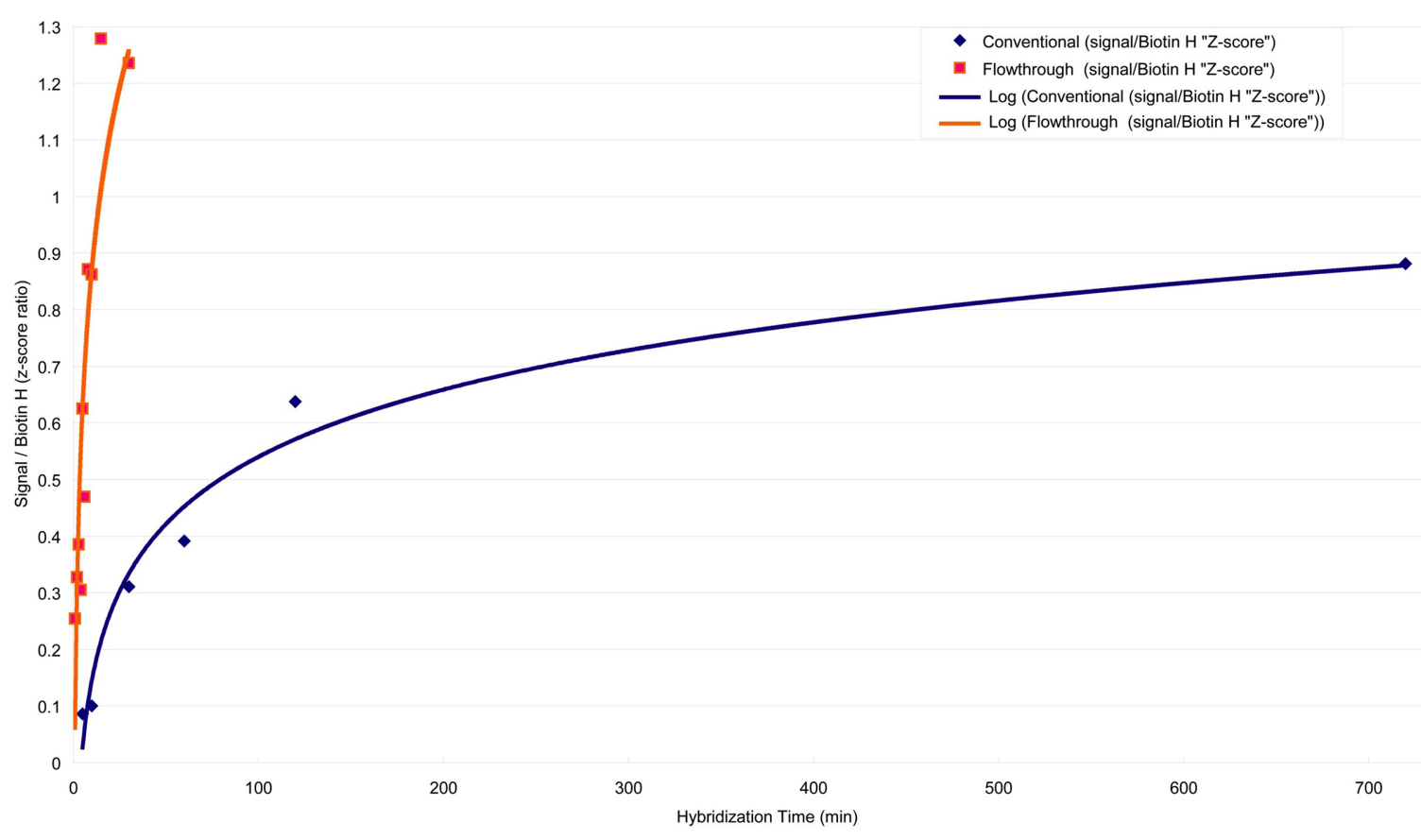

Fig. (2). A plot of Z-score against hybridization time using two different methods. The signal of different hybridization time used in the Flow-through method were plotted against the conventional hybridization method. Flow-through hybridization can achieved a high signal within 15 minutes of hybridization time. A 8 to 10-minutes hybridization time by the Flow-through can achieve the same signal produced by an overnight hybridization using the conventional method.

nential growth of research activity and diagnostic development, a demand for a better hybridization procedure is imperative. However, as in the cases of Southern, dot or slot blotting, it is still a time- and reagent- consuming process, while high-density microarrays are too expensive for clinical implementation. The present study describes the principle of a unique hybridization process, whereby the overall processing time, reagents and steps for hybridization are reduced significantly. Thus the Flow-through method can provide an economical alternative to the nucleic acid diagnostic applications. 
The annealing kinetics of the complementary DNA single strands in solution is a bimolecular collision process which the rate of double strand formation depends on the concentration of the nucleic acid target sequences. In the two-phase conventional membrane based hybridization, the target molecules must move toward the probes that are immobilized in the solid matrix during hybridization. The speed of the diffusion and concentration of the target molecules moving through the solid matrix is the rate limiting step resulting in a long incubation step. The Flow-through method eliminates this diffusion process by directing the target DNA to the complementary capture probes that are immobilized on and inside the membrane pores. In this instance, the target DNA sequence can be facilitated to the annealing process in an active approach manner. Furthermore, this approach facilitates the reaction between the target DNA molecules in a three-dimensional porous environment rather than on planar surfaces. This effectively changes the hybridization action from a passive random diffusion action to an active channeling process, allowing the reactions to be completed in seconds.

At present, conventional or the reverse dot-blot hybridizations are carried out either in glass tubes or plastic bags in a hybridization oven maintained at designated temperatures. The time required to complete the hybridization process ranges from hours to days. The reasons for the slow rate of annealing process are: 1) the need for large reagent volume to cover the whole membrane leading to much lower effective concentration available for binding to the immobilized probe; 2) during the incubation process, the majority of the non-contacted target DNA are self-annealed which reduced the effective concentration of the denatured target DNA. Therefore, a decrease of the remaining target DNA concentration will be expected in the longer hybridization time which reduces the efficiency of the hybridization. 3) Membranes are characteristically porous and DNA molecules of $<500$ bp can readily diffuse through a 0.45 micron membrane. In membrane preparation, a large portion of the 24-30 nucleotide probes will be immobilized inside the matrix pores. In conventional hybridization, only a small portion of the total probes immobilized on the surface of the membrane are accessible for binding to the target DNA molecules.

The Flow-through hybridization method is designed to eliminate all of these intrinsic defects. It generates much higher signal intensities in a very short period of time with much reduced processing time and reagent volumes. In washing, a clean solution front moving forward is anticipated in the Flow-through method each time a new wash is done, making it the most effective washing mechanism possible. Thus besides the higher binding rate resulting in higher intensity, a much cleaner background can also be expected. Consequently in principle, the Flow-through method could be the fastest and cleanest system for producing hybridization assays. Hence it is the most simple and least expensive alternative method for hybridization.

The Flow-through principle and its advantages have been initially demonstrated in the original patent (US Patent $5,741,647)$. This method can be used not only to discriminate non-homologous sequences such as different viruses, it can be used to differentiate from short deletions to single point mutations for thalasemia diagnosis [6]. It was first applied for genotyping identifications of HLA [7], subsequently DNA fingerprinting [8] and proteins (patent pending 2006). Recently, this technology was demonstrated in the use of HBV MYDD typing [9,10] and HPV genotyping [11]. Evidently, the Flow-through technology is a proven technology and is applicable for clinical diagnosis use.

The present study using the principle of Flow-through hybridization and R2-M Flow-through system provides the feasibility evidence for routine application in clinical diagnosis and research purposes. We were able to demonstrate that "low density arrays" for routine DNA analyses was feasible and better than conventional method: (1) as expected the sensitivity was much higher in the Flow-through process than conventional hybridization. The signals generated in 5 to 15 minutes were consistently higher ( 1.5 folds) than those obtained in hours by the conventional technique (Table 2 and Fig. 2); (2) There were no cross hybridization between the target HPV genotype and any of the other 5 different HPV oligonucleotide probes. This indicated that the specificity was high. Indeed, simultaneous analyses of the $33 \mathrm{HPV}$ genotypes have been confirmed and validated in conjunction with the use of the R2-M Flow-through system (data not shown). 3) Our results agreed with the original claims that the Flow-through system resulted in substantial savings in time (up to 20 folds) and in reagents cost ( 8.5 folds) (Table 4). In addition, this relatively inexpensive R2-M Flowthrough system allows all steps from hybridization to signal development to be completed through uncomplicated simple operating procedures.

\section{CONCLUSION}

The array technologies have changed some directions in the past decade. Instead of concentrating on high-density microarray covering thousands of genes for research, many have moved into low-density to cover hundreds or fewer genes or proteins for the diagnostic market. The Reversed Dot Blot (RDB) format for DNA analyses has been widely used for research and in routine clinical diagnoses because it allows simultaneously detect multiple mutations in a single membrane. The present study clearly demonstrated that results acquired from R2-M Flow-through system using Flowthrough hybridization technology can be as efficient at better or equal sensitivity and specificity to the conventional method.

The present R2-M Flow-through system has provided the adequate controlled conditions for rapid and specific nucleic acid hybridization. In addition, the reaction chamber is readily adapted to different format in exchange of accessories. This makes it a versatile platform for other nucleic acids such as Southern, Northern, Dot-Blot, Slot-Blot and Reversed Dot Blot hybridization techniques so far reported on the literature. Western blotting or immuno-blotting analysis on the Flow-through method is suggested. Similar sensitivity and specificity was achieved compared to conventional western blot assay (Personal communication).

\section{REFERENCES}

[1] Maggio A, Giambona A, Cai SP, et al. Rapid and simultaneous typing of hemoglobin S, hemoglobin C, and seven Mediterranean beta-thalassemia mutations by covalent reverse dot-blot analysis: application to prenatal diagnosis in Sicily. Blood 1993; 81(1): 239 42 
[2] Jacobs MV, de Roda Husman AM, van den Brule AJ, et al. Groupspecific differentiation between high- and low-risk human papillomavirus genotypes by general primer-mediated PCR and two cocktails of oligonucleotide probes. J Clin Microbiol 1995; 33(4): 9015.

[3] Gravitt PE, Peyton CL, Apple, RJ, Wheeler CM. Genotyping of 27 human papillomavirus types by using L1 consensus PCR products by a single-hybridization, reverse line blot detection method. J Clin Microbiol 1998; 36(10): 3020-7.

[4] Coutlee F, Gravitt P, Richardson H, et al. Nonisotopic detection and typing of human papillomavirus DNA in genital samples by the line blot assay. The Canadian Women's HIV study group. J Clin Microbiol 1999; 37(6): 1852-7.

[5] Kawasaki ES, Chehab FF. Analysis of gene sequences by hybridization of PCR-amplified DNA to covalently bound oligonucleotide probes. The reverse dot blot method. Methods Mol Biol 1994; 28: 225-36.

[6] Yan XM, Tam JWO. Recent advances in DNA diagnostic technology. Foreign Med Sci 1997; 5: 193-196.
Tam JWO. SNP-based HLA-DP, DR and DQ genotyping analysis by reversed dot blot flow through hybridization. United States patent US 0209253. 2004

[8] Tam JWO. DNA fingerprinting using allelic specific oligonucleotide reversed dot blot (ASO-RDB) flow through hybridization process and device. United States patent US 00794932005. 2005.

[9] Ou ZY, Liu N, Chen CJ, et al. Rapid and accurate genotyping of YMDD motif variants in the hepatitis B virus genome by an improved reverse dot blot method J Clin Microbiol 2005; 43(11): 5685-9.

[10] Zhang R, Deng Y, Muller CP, et al. Determination of hepatitis B virus genotype by flow-through reverse dot blot. J Clin Virol 2007; 39(2): 94-100.

[11] Dai Y, Huang YS, Tang M, et al. Distribution and clinical significance of human papillomavirus subtypes in Shenzhen city, People's Republic of China. Int J Gynecol Cancer 2007 doi:10.1111/j.15251438.2007.01024.x 